\section{Psychosoziale Belastungsfaktoren als Risiko für das Auftreten einer koronaren Herzerkrankung - Eine Bestandsaufnahme unter besonderer Berücksichtigung der KORA-Forschungsplattform}

\author{
Psychosocial Factors as Risk for Coronary Heart Disease - Status with Special \\ Reference to the KORA Platform
}

\author{
K.-H. Ladwig ${ }^{1,2}$ \\ B. Marten-Mittag ${ }^{1,2}$ \\ J. Baumert ${ }^{1,2}$ \\ für die MONICA/KORA-Studien- \\ gruppe*
}

\section{Zusammenfassung}

Gesundheitspsychologische, sozialmedizinische und psychosomatische bzw. psychopathologische Konzepte wurden in der Vergangenheit extensiv auf ihre Fähigkeit zur Vorhersage eines koronaren Ereignisses bei scheinbar herzgesunden Populationen untersucht. Dennoch finden sie in internationalen Algorithmen zur Risikoabschätzung einer koronaren Herzerkrankung gegenwärtig keine Verwendung. In drei MONICA-Surveys (S1-S3) wurde an fast 13000 Teilnehmern im Alter zwischen 25 und 74 Jahren ein breites Spektrum an psychosozialen Daten erfasst, die durch die Weiterführung des Projektes als KORA-Studienplattform mit einer ersten Nachuntersuchung aller Studienteilnehmer im Jahr 1998 einen prospektiven Studienarm bekommen haben und gegenwärtig eine mittlere Beobachtungszeit von sieben Jahren einschließen. Aus der Von-Zerrssen-Beschwerdeliste wurde ein acht Items (Wertebereich 0-24) umfassendes Depressions-Screeninginstrument extrahiert, das über eine ausreichende Validität und Reliabilität verfügt. Männliche Probanden mit hohem Ausmaß an Depressivität haben nach Kontrolle einer großen Zahl von Risikofaktoren ein 1,55fach (95\%-KI: 1,28-1,83, p < 0,0001) erhöhtes Gesamtmortalitätsrisiko und ein 1,36fach (95\%-KI: 1,02 - 1,81, p < 0,035) erhöhtes Risiko für koronare Herzkrankheit (KHK). Frauen weisen höhere Prävalenzen an depressionsbezogenen Werten auf; Depressivität ist bei Frauen jedoch kein messbarer Risikofaktor. Depressivität bei Männern intera-

\section{Abstract}

Psychosocial factors derived from concepts in health psychology and psychopathology are subject of extensive research to assess their power to predict a future coronary artery disease event in apparently healthy subjects. However, bio-behavioural factors have not been implemented in current guidelines of scoring schemes for calculating the risk of coronary events. The presented data were derived from the population-based MONICA Augsburg studies (S1-S3) conducted between 1984 and 1995. The psychosocial data set was available in approximately 13,000 subjects. The KORA follow-up study assessed the vital status for all participants (except for 56 persons) in 1998. Until then, 772 participants (531 men, 241 women) had died. The depressive symptomatology was derived from the von Zerrssen affective symptom check list combining 24 single symptom items with scores ranging from 0 to 3 . Risks of total mortality and myocardial infarction were estimated from Cox proportional hazard ratio (HR) models adjusted for age and survey and multiple risk factors. Male participants with high scores in depression exhibited a significantly increased risk in total mortality (adjusted HR: $1.55 ; 95 \% \mathrm{CI}: 1,28-1,83, \mathrm{p}<0.0001)$ and for fatal and nonfatal coronary events (adjusted HR: 1.36; $95 \% \mathrm{CI}: 1.02-1.81$, $\mathrm{p}<0.035$ ). Female participants reported higher values in depression scores; however, depression was not predictive for subsequent total mortality and fatal and non-fatal events in females.

Anmerkung

*Die MONICA/KORA-Studiengruppe besteht aus: KORA: H.-E. Wichmann (Sprecher), H. Löwel, C. Meisinger, T. Illig, R. Holle, J. John und deren Mitarbeitern, die verantwortlich für das Design und die Durchführung der KORA-Studien sind; MONICA: U. Keil (Studienleiter), A. Döring, B. Filipiak, H. W. Hense, H. Löwel, J. Stieber und den Mitarbeitern, die verantwortlich für das Design und die Durchführung der MONICA-Studien waren.

Institutsangaben

${ }^{1}$ GSF-Forschungszentrum für Umwelt und Gesundheit, Institut für Epidemiologie, Neuherberg

${ }^{2}$ Technische Universität München, Institut und Poliklinik für Psychosomatische Medizin, Med. Psychologie und Psychotherapie des Klinikums rechts der Isar, München

Korrespondenzadresse

Prof. K. H. Ladwig PhD, MD · GSF-National Research Center for Environment and Health, Institute of Epidemiology · Ingolstädter Landstraße 1 · 85764 Neuherberg · E-mail: ladwig@gsf.de

Bibliografie

Gesundheitswesen 2005; 67 Sonderheft 1: S86 -S93 @ Georg Thieme Verlag KG Stuttgart • New York DOI 10.1055/s-2005-858249

ISSN 0949-7013 
giert auf bedeutsame Weise mit Adipositas und erhöhten C-reaktiven Proteinen (CRP). Dem Einschluss psychosozialer Faktoren demonstriert am Beispiel der Depressivität - kommt nicht nur eine zentrale Rolle in der Vorhersage von KHK-Ereignissen zu, sondern er wird in Zukunft auch zur Phänotypisierung von Risiken im Zusammenhang mit der Gen/Umwelt-Diskussion eine wichtige Rolle in der KORA-Studienplattform spielen.

\section{Schlüisselwörter}

Epidemiologie · koronare Herzerkrankung · psychosoziale Risikofaktoren · Depression
Depression in men yielded a significant interaction with obesity and increased levels of C-reactive protein (CRP). The inclusion of psychosocial factors, as demonstrated for depression, is likely to improve prediction of future adverse cardiovascular and total mortality. These factors may also play a crucial role in genotype-phenotype interaction.

\section{Key words}

Epidemiology · coronary heart disease · psychosocial risk factors . depression

\section{Einleitung}

Die Bedeutung komorbider psychopathologischer Zustände bei Patienten mit manifester koronarer Herzkrankheit (KHK) ist wissenschaftlich gut belegt [ 1 -4] und wird gegenwärtig - beispielsweise bei rehabilitativen Behandlungskonzepten der Infarktnachsorge [5] - in therapeutisches Handeln umgesetzt. In der Risikoabschätzung vor dem Manifestwerden einer KHK spielen psychosoziale Risikofaktoren dagegen faktisch keine Rolle.

Zur Vorhersage eines erhöhten Risikos, eine koronaren Herzerkrankung zu erleiden und möglicherweise daran zu versterben, werden gegenwärtig in den internationalen Klassifikationssystemen [6, 7] ausschließlich Algorithmen und Punktesysteme berechnet, die - neben dem Alter und Geschlecht als unbeeinflussbare biologische Determinanten - Parameter des Fettstoffwechsels (LDL-Spiegel, HDL-Spiegel, Triglyzeride), den Zigarettenkonsum, Diabetes mellitus oder erhöhte Nüchternblutzuckerwerte sowie den systolischen Blutdruck einbeziehen. Immerhin weisen sowohl die US Preventive Services Task Force [8] als auch die europäischen Leitlinien zur KHK-Prävention darauf hin, dass ,... negative emotions, including depression, anger and hostility may constitute barriers to preventive efforts ..." [S. 1606] bei Koronarpatienten als auch bei noch nicht erkrankten Hochrisikopersonen. Dennoch ist allen Risikoschätzern gemeinsam, dass psychosoziale Risikofaktoren in den Algorithmen gegenwärtig keine Verwendung finden.

Dabei hat nach unserer Auffassung die Vernachlässigung psychosozialer Risikofakten tatsächlich weniger mit der wissenschaftlichen Datenlage zu tun als mit Gründen, die primär außerhalb wissenschaftlicher Abwägungen liegen. In der folgenden Übersicht möchten wir mit Schwerpunkt auf Auswertestrategien, die im MONICA-Augsburg-Projekt und in der KORA-Plattform möglich geworden sind, zeigen, dass psychosoziale Parameter eine wichtige Funktion in der KHK-Prävention spielen und dass ihnen eine zentrale Rolle in der Vorhersage von KHKEreignissen zukommt.

Zu den psychosozialen Risiken, die in der Vergangenheit extensiv auf ihre Bedeutung zur Vorhersage eines koronaren Ereignisses bei scheinbar herzgesunden Populationen untersucht wurden, zählen gesundheitspsychologische, sozialmedizinische und psychosomatische bzw. psychopathologische Konzepte. Viele dieser Konzepte können mit querschnittlichen, aber auch mit prospek- tiven Ansätzen in den MONICA-Augsburg-Surveys untersucht werden, die in mehreren konsekutiv durchgeführten Querschnittsstudien auf Bevölkerungsniveau und einer prospektiven Nachuntersuchung bei ca. 12888 Teilnehmern (im Alter von 25 bis 74 Jahren) ein international beachtliches Spektrum an psychosozialen Daten erhoben haben (Tab.1).

Tab. 1 Psychosoziale Datensätze in den MONICA-Surveys (S1-S3) (Auswahl)

\begin{tabular}{|c|c|c|c|}
\hline Modul & $\begin{array}{l}\text { S1 } \\
(1984 / 85)\end{array}$ & $\begin{array}{l}S 2 \\
(1989 / 90)\end{array}$ & $\begin{array}{l}\text { S3 } \\
(1994 / 95)\end{array}$ \\
\hline $\begin{array}{l}\text { Selbsteinschätzung des Gesundheits- } \\
\text { zustandes }^{1}\end{array}$ & $x$ & $x$ & $x$ \\
\hline Schlafstörungen ${ }^{2}$ & $x$ & $x$ & $x$ \\
\hline Ärger und Aufregung ${ }^{3}$ & & $x$ & $x$ \\
\hline Von-Zerssen-Beschwerdeliste ${ }^{4}$ & $x$ & $x$ & $x$ \\
\hline seelische Gesundheit (SF-36-Modul) ${ }^{5}$ & & & $x$ \\
\hline Typ-A-Verhalten ${ }^{6}$ & $x$ & $x$ & $x$ \\
\hline Arbeitsplatz-Anforderungsprofil ${ }^{7}$ & $x$ & $x$ & $x$ \\
\hline $\begin{array}{l}\text { interpersonelle Beziehungen am } \\
\text { Arbeitsplatz }^{8}\end{array}$ & $x$ & $x$ & $x$ \\
\hline sozialer Netzwerk-Index ${ }^{9}$ & $x$ & $x$ & $x$ \\
\hline \multicolumn{4}{|c|}{$\begin{array}{l}\text { zwei Items nach Kaplan GA, Camacho T. (1984) [36]; }{ }^{2} \text { zwei Items (MONICA } \\
\text { core design); }{ }^{3} 11 \text {-stufige Ärgerskala, modifiziert nach Spielberger et al. (1984) } \\
\text { [37]; }{ }^{4} 24 \text { Items umfassende Beschwerdeliste, 5fach skaliert [38]; }{ }^{5} \text { Items um- } \\
\text { fassende, 6fach skaliertes Mental-Health-Modul aus dem SF } 36 \text { (Ware 1994) } \\
\text { [39]; }{ }^{6} 10 \text { vierfach skalierte Items der Framingham-Typ-A-Skala (Jenkins et al. } \\
\text { 1971) [40]; }{ }^{7} 11 \text { vierfach skalierte Items (Karasek et al. 1982) [41]; }{ }^{8} \text { allgemeine } \\
\text { Zufriedenheit am Arbeitsplatz (Siegrist et al. 1982) [42]; }{ }^{9} \text { Berkman-Index (Ala- } \\
\text { meda County Study 1978) [43-45] }\end{array}$} \\
\hline
\end{tabular}

\section{Gesundheitspsychologische Ansätze}

Als Beispiel für einen gesundheitspsychologischen Ansatz hat sich die subjektive Selbsteinschätzung des Gesundheitszustandes (self perceived health) als ein erstaunlich valider Prädiktor für KHK herausgestellt [9]. Heidrich et al. untersuchten 1521 Männer und 1498 Frauen im Alter von 35-64 Jahren aus dem MONICA-Survey 1984/85 (S1) [10]. Nach einer Nachverfolgungszeit von 11 Jahren wurde der medizinische Status einschließlich der Risikofaktoren dieser Population untersucht. Die Untersucher unterteilten die Probanden in solche, die ihre Gesundheit als mäßig bis schlecht eingeschätzt hatten, und solche, die ihre Gesundheit als gut bis exzellent bewertet hatten. Sie errechneten 
dann das relative Sterberisiko mit Hilfe von adjustierten Hazards Rate Ratios (HRRs) und fanden, dass das Gesamtmortalitätsrisiko für Männer bei einer HR von 1,5 (95\%-KI: 1,1 - 2,2) für die negative Selbsteinschätzung des Gesundheitszustandes gegenüber einer guten Selbstwahrnehmung lag. Für Frauen konnte dagegen kein erhöhtes Risiko gesichert werden (HRR $=1,1,95 \%-\mathrm{KI}$ : $0,7-1,9)$. Die Effekte für kardiovaskulären Tod als Endpunkt gingen in die gleiche Richtung, waren jedoch nicht signifikant.

\section{Sozialmedizinische Ansätze}

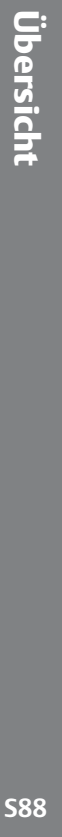

Sozialmedizinische und sozialpsychologische Forschungsansätze untersuchten den Einfluss aversiver Lebensumstände (wie Armut, Zugehörigkeit zur Unterschicht; Arbeitslosigkeit, akute und chronische [Dis-]Stress-Erfahrungen am Arbeitsplatz oder in der Partnerschaft), schwerwiegende Lebensereignisse (life events) wie Scheidung, Tod des Partners oder der Verlust des Arbeitsplatzes, aber auch chronische Belastungen durch soziale Isolation und Einsamkeit.

Die Bedeutung von psychosozialem Stress als KHK-Risikofaktor wurde durch die kürzlich publizierten Ergebnisse der großformatigen Interheart Study [11], die insgesamt fast 15000 Fälle und eine ebenso große Zahl von Kontrollpatienten aus 52 Ländern einschloss, eindrucksvoll untermauert. Es konnte gezeigt werden, dass Stress in der Vorhersagekraft - gemessen in der Höhe der Odds Ratios - nach Rauchen und einem Parameter des Fettstoffwechsels rangierte, aber noch vor Diabetes, Bluthochdruck und Adipositas mit KHK assoziiert ist [12].

Eine Reihe von epidemiologischen Studien belegt, dass soziale Isolation mit einem hohen prospektiven Risiko von Herz-Kreislauf-Krankheiten (HKK) und dadurch bedingter Mortalität verbunden ist. Warum dies so ist, ist allerdings nicht ausreichend geklärt. So legen einige Untersuchungen nahe, dass der sozialpsychologische Faktor „soziale Isolation“ indirekter Indikator für höheres Alter und niedriges Einkommen ist und damit ausreichend erklärt wird [13], während andere Arbeiten dafür sprechen, dass soziale Isolierung ein hohes Ausmaß an psychologischem Dis-Stress, Schweregrad der Erkrankung und negativen demographischen Faktoren abbildet.[14] Brummett et al. [15] konnten kürzlich an einer Gruppe von 430 Koronarpatienten zeigen, dass ein hohes Ausmaß an sozialer Isolation mit einem signifikant erhöhten Mortalitätsrisiko verbunden war, dass dieses Risiko aber unabhängig von demographischen Faktoren, dem Schweregrad der Erkrankung und dem Ausmaß an psychologischem „Dis-Stress“ war. Diese Arbeit bestätigt den negativen Einfluss des Faktors auf den Krankheitsverlauf, wirft damit aber die Frage auf, warum die Betroffenen an sozialer Isolation sterben. Hierzu werden in Kürze Ergebnisse aus dem MONICA/KORA-Projekt vorliegen.

\section{Psychosomatische Konzepte}

Psychosomatische Konzepte reflektieren objektive aversive $\mathrm{Zu}$ stände wie Arbeitsplatzverlust, Belastungen in Beruf und Partnerschaft etc. als subjektive Befindlichkeit, gehen aber als Parameter einer negativen Affektivität weit darüber hinaus, weil sie die individuelle Lerngeschichte und die inhärenten Bewältigungsmöglichkeiten der Betroffenen mit einschließen. Als KHKRisikofaktoren wurden bislang z. B. Angst und Depression, Panik, chronischer Ärger, Feindseligkeit, Zynismus, hyperaktives Verhalten sowie Hilflosigkeit untersucht.

Kubzansky et al. [16] fanden in einer Kohorte von 1758 herzgesunden Männern, dass andauernde Besorgnis (insbesondere über soziale Lebensaspekte) mit einem 1,5fach erhöhten Risiko für das Erstauftreten einer koronaren Herzerkrankung assoziiert war. Haines und Mitarbeiter [17] untersuchten bei 1457 Männern im Alter von 40 bis 64 Jahren aus der Northwick-ParkHeart-Studie den Einfluss von phobischer Angst auf das Risiko, einen tödlichen Myokardinfarkt zu erleiden. Die Ergebnisse zeigten, dass mit ansteigender phobischer Angst nachfolgende KHKEreignisse auch nach Kontrolle klassischer kardiovaskulären Risikofaktoren (wie etwa Alter, Zigarettenrauchen, Bluthochdruck und Cholesterinkonzentration) signifikant vorhergesagt werden konnten. Phobische Angst war wiederum nicht mit weiteren Todesursachen assoziiert, so dass die Autoren auf einen spezifischen Zusammenhang zwischen phobischer Angst und nachfolgender KHK (insbesondere tödlichen Myokardinfarkten) schlossen.

Zu einem ähnlichen Ergebnis kamen Kawachi et al. [18], die den Einfluss von phobischer Angst auf die Vorhersage eines KHK-Ereignisses bei 33999 herzgesunden Männern im Alter von 42 - 77 Jahren aus der Health Professional Study untersuchten. Sie fanden, dass das altersadjustierte relative Risiko, einen tödlichen akuten Myokardinfarkt zu erleiden, in der Gruppe der Untersuchten mit dem höchsten Ausmaß phobischer Angst im Vergleich zu der Gruppe mit niedrigster Angstausprägung ein relatives Risiko von 3,01 (95\%-KI: 1,31 - 6,9) aufwies. Ein Dosis-Wirkungs-Effekt konnte gesichert werden ( $p$-Trend $=0,002$ ).

Zustände von akuter Angst führen zu ausgeprägten körperlichen Symptomen, die Ausdruck einer sympathikotonen Hyperregulation sind. Der psychobiologische Nutzen der Angst liegt in der autonom regulierten Vorbereitung (Bereitschaftsstellung) einer „fight or flight“(Kampf-oder-Flucht)-Reaktion. Menschen im Zustand chronischer Angst und Besorgtheit befinden sich in einem dauerhaft erhöhten Reaktionsmodus, der mit überschießenden Kreislauf- und Blutdruckreaktionen sowie mit einer ingeschränkten Flexibilität und Variabilität der autonomen Regulation, d.h. mit unbalancierter sympathikotoner bzw. einer nicht inhibierenden parasympathischen Kontrolle, einhergeht. Diese Modellvorstellung könnte erklären, warum chronische Angst und Besorgnis ein Risikofaktor für das Auftreten einer koronaren Herzerkrankung ist.

\section{Prävalenz und Klinik der Depression}

Depressive Erkrankungen sind häufig, vielfach chronisch und kostspielig [19]. Häufigkeitsschätzungen auf der Basis US-amerikanischer, bevölkerungsbezogener Surveys gehen von einer Punktprävalenz (innerhalb der letzten vier Wochen) von 1,8\% bis 3,3\% und einer Lebensprävalenz bei Erwachsenen von 4,9\% bis $17,1 \%$ aus [20]. Neueste Surveydaten sprechen für eine Lebenszeitprävalenz von 16,6\% (95\%-KI: 15,1 - 17,2) [21]. 


\section{Klinisches Bild einer depressiven Episode}

Bei den typischen Episoden einer depressiven Erkrankung leidet der betroffene Patient unter einer gedrückten Stimmung und einer Verminderung von Antrieb und Aktivität. Die Fähigkeit zur Freude, das emotionale Anteilnehmen sowie das Interesse und die Konzentration sind vermindert. Ausgeprägte Müdigkeit kann nach jeder kleinsten Anstrengung auftreten. Der Schlaf ist meist gestört, der Appetit vermindert. Selbstwertgefühl und Selbstvertrauen sind fast immer beeinträchtigt. Sogar bei der leichten Form kommen Schuldgefühle oder Gedanken über die eigene Wertlosigkeit vor. Die gedrückte Stimmung verändert sich von Tag zu Tag wenig, reagiert nicht auf Lebensumstände und wird in der Regel von „Defizit-Symptomen“ wie Verlust an Interessen, der Freude, des Appetits und der Libido begleitet. Hinzu kommen Symptome wie Früherwachen, Morgentief, deutliche psychomotorische Hemmung oder Agitiertheit. Bei einer schweren depressiven Episode sind Halluzinationen, Wahnideen, psychomotorische Hemmung oder ein Stupor so schwer ausgeprägt, dass alltägliche soziale Aktivitäten unmöglich sind und Lebensgefahr durch Suizid und mangelhafte Flüssigkeitsund Nahrungsaufnahme bestehen kann.

Konsistente Forschungsergebnisse zu den gesundheitlichen Folgen von Episoden unipolarer Depression haben zeigen können, dass die Depression in der Primärversorgung eine kostspielige, zu erheblichen Gesundheitseinschränkungen (bis hin zu letalen Konsequenzen) führende Erkrankung ist, die dazu als „unterdiagnostiziert und unterbehandelt“ [22] gilt. Nach den Ergebnissen der „WHO-Global Burden of Disease“-Studie gehört eine unipolare schwere depressive Episode zu den führenden Ursachen für Gesundheitsstörungen weltweit und das Maß „burden of disease“, definiert als ein kombinierter Effekt von Mortalität und Gesundheitsstörung, setzt die Depression mit den negativen Konsequenzen der koronaren Herzerkrankung gleich [23].

\section{Depression als KHK-Risikofaktor}

Episoden manifester Depression, eine andauernde depressive Verstimmung und Symptome vitaler Erschöpfung gehören zu einem gemeinsamen Symptom-Cluster, dessen Bedeutung als Risikofaktor in der Vorgeschichte einer koronaren Herzerkrankung eine hohe Evidenz besitzt. Drei aktuelle Metaanalysen [24-26], die jeweils mehr als 10 bevölkerungsbezogene prospektive Studien zur Grundlage hatten, konnten übereinstimmend mit nur einer Baseline-Messung depressiver Symptome nach Kontrolle aller klassischen HKK-Risikofaktoren ein signifikant erhöhtes Mortalitätsrisiko nachweisen, das mit einer adjustierter OR von 1,64 im Vorhersagebereich der klassischen Risikofaktoren liegt.

\section{Klinisches Bild der KHK-typischen Depressivität}

Die Klinik der depressiven Komorbidität bei Risikopatienten und Patienten mit manifester KHK hat wenig gemein mit den Kernsymptomen einer schweren depressiven Episode, die mit einer psychotischen Symptomatik verschwistert ist. Das Symptombild bei KHK-Patienten zeichnet sich charakteristischerweise eher durch ein neurasthenisches, dysthymes Erscheinungsbild aus, zu dessen Hauptcharakteristika die Klage über vermehrte Müdigkeit nach geistigen Anstrengungen, häufig verbunden mit abnehmender Arbeitsleistung oder Effektivität bei der Bewältigung täglicher Aufgaben, gehört. Hinzu kommen häufig Gefühle körperlicher Schwäche und Erschöpfung nach nur geringer Anstrengung und der Unfähigkeit, sich zu entspannen. Die geistige Ermüdbarkeit wird typischerweise als unangenehmes Eindringen ablenkender Assoziationen oder Erinnerungen beschrieben, als Konzentrationsschwäche und allgemein ineffektives Denken. Reizbarkeit, Freudlosigkeit, Niedergeschlagenheit, pessimistische Zukunftserwartung, Schlafstörungen und Angst sind häufig.

Eine beeindruckend große Zahl von bevölkerungsbezogenen, prospektiv angelegten Kohortenstudien konnte übereinstimmend bei zunächst Gesunden ein erheblich erhöhtes Risiko sichern, eine koronare Herzerkrankung zu erleiden, wenn diese Probanden Symptome einer andauernd depressiven Stimmungslage zeigten. Rugulis [24] konnte seine Metaanalyse auf ein effektives n von etwa 38000 in prospektive bevölkerungsbezogene Studien eingeschlossenen Probanden stützen. Darin nicht eingeschlossen waren die Daten aus den MONICA/KORA-Surveys sowie weiterer zwischenzeitlich publizierter Studien [27], so dass die Evidenz der prospektiven Bedeutung einer depressiven Stimmungslage mittlerweile mehr als 60000 Probanden einschließt.

Zu den offenen Forschungsfragen zählt der Einfluss des Geschlechts auf die Bedeutung einer Depression als KHK-Risikofaktor. Allgemein ist bekannt, dass Frauen häufiger über depressive Symptome berichten als Männer; unklar ist jedoch, ob die Symptomatik die gleichen malignen Auswirkungen auf Gesamtmortalität und KHK-Ereignisse hat wie bei männlichen Betroffenen. Wenig ist auch über die Wirkweise und den pathophysiologischen Wirkmechanismus dieser offenkundig „letalen Expositions-Variablen“ [26] bekannt. Zwar existieren eine Reihe von wichtigen Konzepten über diesen Zusammenhang sowie erste experimentelle und klinische Daten, dennoch ist nach heutigem Stand des Wissens die Verursachungsfrage ungeklärt. Epidemiologische Untersuchungen wurden bislang hierzu nicht durchgeführt.

\section{Depressionsforschung im MONICA/KORA-Projekt}

Trotz einer Vielzahl von Instrumenten zur Messung von psychosozialen Variablen, die in den MONICA-Surveys eingesetzt wurden (Tab.1), sah das Studienprotokoll der Surveys S1-S3 (1984/85 bis 1994/95) keine explizite Depressionsmessung vor. Es gelang aber, aus der 24 Items umfassenden Von-Zerssen-Beschwerdeliste mit Hilfe einer Faktorenanalyse (varimax rotation) eine Fünf-Faktorenlösung zu extrahieren, die einen Faktor mit acht Items generierte, der inhaltlich dem klinischen Bild eines Erschöpfungs- und Depressionssyndroms, so wie es typisch für KHK-Patienten ist, zugeordnet werden konnte. Die Depressionsskala (Wertebereich zwischen 0-24) war normalverteilt und erwartungsgemäß zeigten Frauen höhere Werte, was zu einer geschlechtsspezifischen Kategorisierung führte. Die Parameter waren alle hochsignifikant korreliert ( $p<0,0001)$. Die interne Konsistenz war mit einem Cronbach's Alpha von 0,88 sehr gut und die Retest-Reliabilität nach 4 Jahren war ausgesprochen hoch $(r=0,83)$. Messungen zur konkurrierenden Validität sowie zur Konstrukt- und prädiktiven Validität erbrachten Ergebnisse, die den Wert der Skala als Messinstrument sichern konnten (Ein- 
zelheiten in [28]). Die so gewonnene Depressionsskala wird gegenwärtig in einer Vielzahl von Einzeluntersuchungen eingesetzt. Bislang konnten die folgenden Ergebnisse erzielt werden:

1. Probanden mit erhöhten Depressionswerten weisen gleichzeitig erhöhte Blutdruck- und Cholesterinwerte (besonders ausgeprägt bei Frauen) auf; sie bewegen sich körperlich deutlich weniger und leiden häufiger an Diabetes mellitus (Tab. 2). Adipositas und Rauchen haben hingegen keinen univariat signifikanten Einfluss auf Depressivität. Erwartungsgemäß ist Depressivität mit einer Vielzahl von Parametern einer negativen Affektivität (wie z.B. pessimistische Selbstwahrnehmung des Gesundheitszustandes sowie Einund Durchschlafstörungen) assoziiert (s. Abb.1).

Tab. 2 Klassische Risikofaktoren bei Männern $(n=6,103)$ und Frauen $(n=6,584)$ im Alter von 25-74 Jahren, getrennt nach Depressivität (gepoolte Daten aus MONICA S1-S3)

\begin{tabular}{|c|c|c|c|c|c|}
\hline \multirow[t]{2}{*}{ Männer } & \multicolumn{2}{|c|}{$\begin{array}{l}\text { nicht depressiv } \\
\mathrm{n}=4259\end{array}$} & \multicolumn{2}{|c|}{$\begin{array}{l}\text { depressiv } \\
\mathrm{n}=1844\end{array}$} & \multirow[t]{2}{*}{ 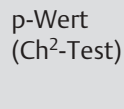 } \\
\hline & $\mathrm{n}$ & $\%$ & $\mathrm{n}$ & $\%$ & \\
\hline Übergewicht (BMI > 30 kg/m²) & 787 & 19 & 321 & 18 & 0,355 \\
\hline Gesamtcholesterin > $240 \mathrm{mg} \mathrm{IL}$ & 1743 & 41 & 804 & 44 & 0,040 \\
\hline regelmäßiger Raucher & 1254 & 29 & 531 & 29 & 0,610 \\
\hline Bluthochdruck & 979 & 23 & 515 & 28 & $<0,001$ \\
\hline körperliche Aktivität & 2042 & 48 & 704 & 38 & $<0,001$ \\
\hline Diabetes mellitus & 135 & 3 & 119 & 7 & $<0,001$ \\
\hline \multirow[t]{2}{*}{ Frauen } & \multicolumn{2}{|c|}{$\begin{array}{l}\text { nicht depressiv } \\
\mathrm{n}=4069\end{array}$} & \multicolumn{2}{|c|}{$\begin{array}{l}\text { depressiv } \\
\mathrm{n}=1777\end{array}$} & $\begin{array}{l}\text { p-Wert } \\
\left(\chi^{2} \text {-Test }\right)\end{array}$ \\
\hline & $n$ & $\%$ & $\mathrm{n}$ & $\%$ & \\
\hline Übergewicht (BMI > 30 kg/m²) & 750 & 19 & 314 & 18 & 0,533 \\
\hline Gesamtcholesterin > $240 \mathrm{mg} \mathrm{IL}$ & 1473 & 37 & 727 & 42 & $<0,001$ \\
\hline regelmäßiger Raucher & 751 & 19 & 350 & 20 & 0,265 \\
\hline Bluthochdruck & 692 & 17 & 383 & 22 & $<0,001$ \\
\hline körperliche Aktivität & 1686 & 42 & 602 & 34 & $<0,001$ \\
\hline Diabetes mellitus & 105 & 3 & 77 & 4 & $<0,001$ \\
\hline
\end{tabular}

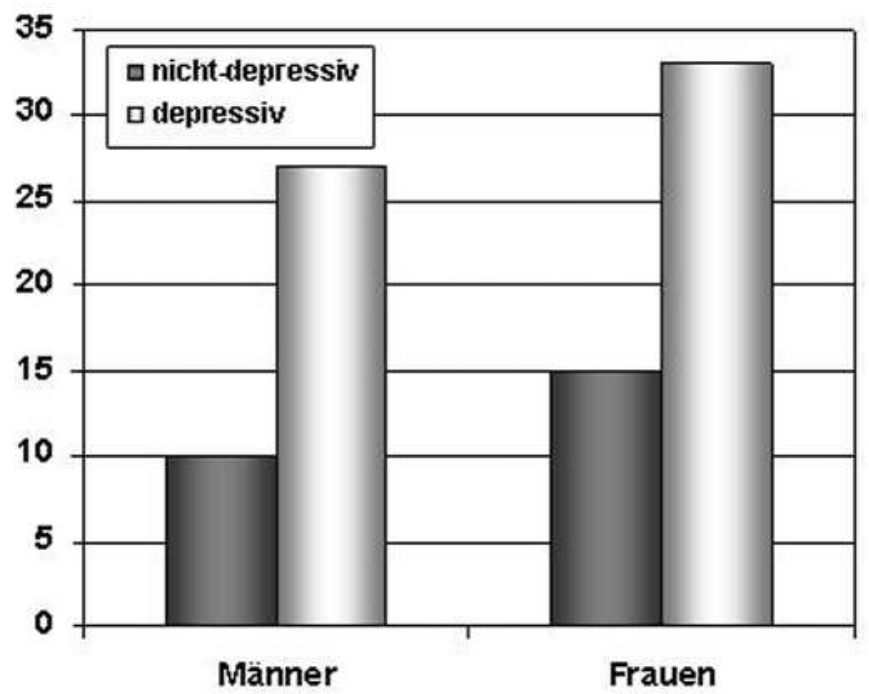

Abb. 1 Häufigkeit von Durchschlafstörungen (Prozentangabe) bei Männern und Frauen, getrennt nach Depressivität $(n=12888)$ (gepoolte Daten aus MONICA S1-S3).
2. Um zu untersuchen, ob sich die maligne Bedeutung einer depressiven Stimmungslage als unabhängiger Prädiktor sowohl für Gesamtmortalität als auch für das Erstauftreten einer koronaren Herzerkrankung bestätigen lässt, konnten die Basisdaten von 5984 Männern sowie von 5622 Frauen aus S1-S3 im Alter von 45 bis 74 Jahren, deren Schicksal über einen durchschnittlichen Beobachtungszeitraum von 7 Jahren nachverfolgt werden konnte, ausgewertet werden. Bei den Männern wurden insgesamt 434 Todesfälle und 202 tödliche und nichttödliche KHK-Ereignisse, bei den Frauen 173 Todesfälle und 50 KHK-Ereignisse festgestellt.

3. Die Vorhersagemodelle mit Hilfe von Cox proportional hazard ratios (HRs) ergaben - nach Kontrolle der Risikofaktoren Rauchen, Adipositas, systolischer Blutdruck, Gesamtcholesterin, Alkoholkonsum, Ausbildungsjahre als Schichtindikator und körperliche Bewegung - bei Männern eine HR von 1,55 (95\%-KI: 1,28-1,83, p <0,0001) für Gesamtmortalität und eine HR von 1,36 (95\%-KI: 1,02 - 1,81, p < 0,035) für KHK-Inzidenz. Dagegen konnten bei Frauen keine signifikanten Ergebnisse erzielt werden: HR 0,94 (95\%-KI: 0,69-1,30, p=0,719) für Gesamtmortalität und eine HR von 1,16 (95\%-KI: 0,65-2,06) für KHK-Inzidenz. Nach diesen Daten sieht es so aus, dass Männer weniger häufig Symptome einer depressiven Stimmungslage offenbaren, doch wenn sie dies tun, sind die Konsequenzen deutlich gravierender.

4. Warum Depressivität mit einem erhöhten kardiovaskulären Ereignisrisiko assoziiert ist, ist bislang ungeklärt. Möglicherweise spielen proinflammatorische Prozesse hierbei eine bedeutsame Rolle, da Akute-Phase-Proteine und proinflammatorische Zytokine sowohl im atherosklerotischen Grundprozess als auch bei affektiven Stresszuständen freigesetzt werden (Übersicht in [29]). Bei einer Kohorte von 3021 gesunden Männern aus S1-S3 im Alter von 45 bis 74 Jahren aus der MONICA-Augsburg-Kohortenstudie wurde der synergistische Effekt von erhöhtem C-reaktiven Protein und Depression untersucht [30]. Hierbei wurde die Gesamtgruppe in Männer mit keiner oder niedriger Ausprägung an Depressivität $(n=2,035)$ und solche mit hoher Ausprägung an Depressivität ( $n=986)$ unterteilt. Erhöhtes CRP hat im Allgemeinen ein zweifach erhöhtes KHK-Risiko zur Folge. Es zeigte sich aber in dieser Untersuchung, dass in der Niedrigdepressionsgruppe erhöhtes CRP keine Vorhersagekraft besaß, während in der Depressionsgruppe CRP eine HR von 2,7 (HR: 2,69; 95\%-KI: 1,32-5,47) aufwies und damit belegte, dass beide Parameter miteinander interagieren [28].

5. Wie komplex die Wechselwirkung zwischen CRP und Depressivität ist, zeigte eine Untersuchung, die in einem querschnittlichen Untersuchungsdesign bei übergewichtigen 726 (23\%) Männern (Bodymass-Index BMI $\geq 30 \mathrm{~kg} / \mathrm{m}^{2}$ ) durchführt wurde, die aus einer Grundgesamtheit von 3204 Männern im Alter von 45 bis 74 Jahren aus der MONICA-Studie (S1-S3) stammten [31]. In dieser Untersuchung zeigte sich, dass ein hohes Ausmaß von Depressivität den CRPSpiegel nur bei den Probanden erhöhte, die einen BMI $>30 \mathrm{~kg} / \mathrm{m}^{2}$ aufwiesen. Bei den normalgewichtigen Männern war das CRP unauffällig über drei Depressionsgruppen (normal, leicht und deutlich erhöht) verteilt. 
6. Eine depressive Stimmung ist für den Betroffenen mit Leid, Lebensqualitätsverlust und Einschränkung seines sozialen Handlungsspielraums verknüpft. Bei einer Untersuchung über Merkmale von Rauchern, die bereit waren, das Rauchen aufzugeben, im Vergleich zu Rauchern, die völlig unwillig waren, ihr Rauchverhalten $\mathrm{zu}$ ändern, zeigte sich, dass eine depressive Stimmungslage aber auch eine psychobiologisch positive Funktion haben kann. Unter 3229 regelmäßigen Rauchern im Alter von 25 bis 74 Jahren aus der MONICA-Studie (S1-S3) waren $23 \%$ völlig unmotiviert und rund $18 \%$ erfüllten die strengen Kriterien für „aufgabewillig“. Eine depressive Stimmungslage trug bei Männern zur Zugehörigkeit zur letzteren Gruppe bei (OR=2,5, 95\%-KI: 1,6-3,8) [32].

\section{Zukünftige psychosoziale KORA-Forschungsschwerpunkte}

Die KORA-Studie 2004/2005 (F3), ein Follow-up des MONICASurveys S3 aus den Jahren 1994/95, wird Mitte 2005 abgeschlossen sein. Die F3-Untersuchung mit einem Schwerpunkt in der Phänotypisierung negativer Affektivität wird mit aktuellen, standardisierten psychodiagnostischen Instrumenten die Messung einer depressiven Symptomatik und negativen Affektivität sicherstellen; weitere Schwerpunkte sind die gesundheitliche Lebensqualität vor allem im Rahmen gesundheitsökonomischer Analysen zum Übergewicht sowie die Rolle sozialer Netzwerke für HKK (Tab. 3). Hierzu werden Daten sowohl im Interviewteil als auch im Selbstausfüllbogen (SAF) erhoben. Die im Interviewteil erhobenen Daten erlauben eine Abschätzung der Depressivität nach den internationalen DSM-IV- bzw. ICD-10-Kriterien, während die im SAF erhobenen Daten depressive Verstimmung auch in Kombination mit sozialer Inhibition, gesundheitlicher Lebensqualität sowie sozialen Beziehungen erfassen.

Die Auswertung der F3-Studie wird sich - zunächst auf querschnittlichem Niveau - in der Depressionsforschung auf Fragestellungen konzentrieren, die dazu beitragen sollen, den Pathomechanismus einer depressiven Symptomatik in Bezug auf kardiovaskuläre Ereignisse aufzuklären. Hierzu sollen Arbeits-

Tab. 3 Psychosoziale Datensätze im KORA-Survey 2004/05 (F3)

1. Brief Patient Health Questionnaire (Brief PHQ) Modul Depression [46]

2. Fragebogen zum Gesundheitszustand (SF-12) [47, 48]

3. Hospital Anxiety and Depression Scale (HADS-D) [49]

4. Beschwerdeliste Von-Zerrsen-Skala (VZ) [38]

5. Framingham-Type-A-Verhaltensskala (TABP) [40]

6. soziale Inhibition und negativer Affekt (Typ-D-)Skala [50]

7. Fagerström-Test for Nicotine Dependence (FTND-G) [51]

8. Multidimensional Health Locus of Control (MHLC-Skalen) $[52,53]^{2}$

9. sozialer Netzwerk-Index (SNI - Berkman-Index) [43-45] $]^{2}$

10. Fragebogen zur sozialen Unterstützung (F-SozU-K14) [54 $]^{2}$

11. Sozialkapital: Social Trust (RECALL-Items nach [55]) ${ }^{2}$

${ }^{1}$ Dieses Instrument wird im Teilprojekt Gesundheitsökonomie des Übergewichts eingesetzt (Federführung: Dr. Jürgen John, GSF-Institut für Gesundheitsökonomie und Management im Gesundheitswesen). 2 Diese Instrumente werden im Teilprojekt Soziale Beziehungen, Sozialkapital und kardiovaskuläre Erkrankungen eingesetzt (Federführung: Dr. Thomas von Lengerke, GSF-Institut für Gesundheitsökonomie und Management im Gesundheitswesen). hypothesen untersucht werden, von denen einige im Folgenden genannt werden:

\section{Verhaltensaspekt}

Depressive Patienten sind medizinisch unterversorgt. Sie berichten über signifikant mehr Beschwerden und sonstige Komorbidität, weisen aber gleichzeitig ein geringeres Ausmaß an Inanspruchnahme medizinischer Dienstleistungen auf. Depression geht mit einer Minderung des Antriebes und der Motivationslage einher - die Compliance mit präventivmedizinischen Maßnahmen ist daher signifikant gegenüber Nichtdepressiven verringert und selbstschädigendes Verhalten (Alkoholmissbrauch, körperliche Inaktivität etc.) messbar häufiger.

\section{Autonome Regulation}

Patienten mit depressivem Syndrom weisen eine gestörte Balance des sympatikotonen mit dem parasympathischen Nervensystem auf. Klinische Untersuchungsreihen haben dies mit Untersuchungen der Herzfrequenz-Variabilität (HFV) zeigen können. Im KORA-Projekt werden HFV-Messungen (unter der Federführung von Siegfried Perz) auf epidemiologischem Niveau zur Verfügung stehen, anhand derer diese Fragestellung weiter untersucht werden soll.

\section{Chronischer Stress}

Depressive Stimmungslagen stellen für die Betroffenen Zustände chronischer Überforderung (chronic stress) dar. Solche Zustände chronischen Stresses sind assoziiert mit bedeutsamen Veränderungen in der Interaktion zwischen arteriellem Blutsystem und Gefäßwand. Zu aussichtsreichen Kandidaten gehören hier Parameter einer Aktivierung der Gerinnungskaskade mit einer eingeschränkten fibrinolytischen Aktivität [33].

\section{Neuroimmunologie}

Chronische Belastungen führen zu neuronalen und endokrinen Funktionsänderungen, aber auch zu einer Aktivierung des Immunsystems. So sind schwere depressive Episoden, postvirale Depressionen und dysthyme Zustände mit erhöhten Plasmakonzentrationen von proinflammatorischen Zytokinen [34] assoziiert. Im Tiermodell führt die Freisetzung von Interleukinen zu der Auslösung von Krankheitsverhalten [35], das durch Anhedonia, Gewichtsverlust, Einschränkung des sozialen Handlungsspielraums, Schlafstörungen, Hyperalgesie etc. charakterisiert ist. Denkbar ist, dass die durch chronifizierte Belastungen stimulierte Aktivierung des Immunsystems zu einer stressinduzierten Zytokinspiegelerhöhung im Plasma führt, die wiederum a) zu einer weiteren Aktivierung des autonomen Systems führt und b) Inflammationsprozesse auslöst, beschleunigt oder verstärkt, die sich in der Gefäßwand abspielen.

Die Feststellung psychopathologischer Auffälligkeiten dient nicht nur Fragestellungen, die sich mit der Verursachung und prospektiven Bedeutung verhaltenswirksamer und affektiver Risikofaktoren beschäftigen, sondern auch zur Phänotypisierung von Risiken, die im Zusammenhang mit der Gen/Umwelt-Diskussion virulent werden. Psychische Faktoren stellen in diesem Zusammenhang Bedingungen der „inneren Umwelt“ dar, die möglicherweise moderierend auf den Ursache-Wirkungs-Zusammenhang einwirken. 
${ }^{18}$ Kawachi I, Colditz GA, Ascherio A et al. Prospective study of phobic anxiety and risk of coronary heart disease in men. Circulation 1994; 89: $1992-1997$

Die Untersuchungen zu psychosozialen und neurologischen Fragestellungen in MONICA/KORA wurden gefördert durch die GSF und das BMBF - Bundesministerium für Forschung und Technologien (01 EG 9405/8, 01 E6 9404) und DFG - Deutsche Forschungsgemeinschaft (BE1996/1-1).

Der Artikel nimmt besonders Bezug auf folgende Beiträge dieses Sonderhefts von „Das Gesundheitswesen”: [56-63].

\section{Literatur}

${ }^{1}$ Ladwig KH, Roth R, Lehmacher W et al. The impact of post-infarction depression on six month survival after acute myocardial infarction. Circulation 1992; 86: 865

${ }^{2}$ Ladwig KH, Röll G, Roth R et al. Contribution of post-infarction depression to an incomplete recovery six months after acute myocardial infarction. Lancet 1994; 343: 20-23

${ }^{3}$ Herrmann-Lingen C, Buss U. Angst und Depressivität im Verlauf der koronaren Herzkrankheit. Reihe Statuskonferenz Psychokardiologie. Frankfurt/M: VAS-Verlag, 2002

${ }^{4}$ Barth J, Schumacher M, Herrmann-Lingen C. Depression as a risk factor for mortality in patients with coronary heart disease: A metaanalysis. Psychosom Med 2004; 66 (6): 802 - 813

${ }^{5}$ Rafanelli C, Roncuzzi R, Finos L et al. Psychological Assessment in Cardiac Rehabilitation. Psychother and Psychosom 2003; 72: $343-349$

${ }^{6}$ Hense HW, Schulte $\mathrm{H}$, Lowel $\mathrm{H}$ et al. Framingham risk function overestimates risk of coronary heart disease in men and women from Germany -results from the MONICA Augsburg and the PROCAM cohorts. Eur Heart J 2003; 24: 937 - 945

${ }^{7}$ Empana JP, Ducimetiere P, Arveiler D et al. Are the Framingham and PROCAM coronary heart disease risk functions applicable to different European populations? The PRIME Study. Eur Heart J 2003; 24: $1903-1911$

${ }^{8}$ De Backer G, Ambrosioni E, Borch-Johnsen K et al. American Heart Association. American College of Cardiology. European guidelines on cardiovascular disease prevention in clinical practice. Third Joint Task Force of European and other Societies on Cardiovascular Disease Prevention in Clinical Practice. Atherosclerosis 2004; 173: 381 - 91

${ }^{9}$ Idler EL, Benyamini B. Self rated health and mortality review of twenty-seven community studies. J Health Soc Behav 1997; 38: 21 - 37

${ }^{10}$ Heidrich J, Liese AD, Löwel $\mathrm{H}$ et al. Self-rated health and its relation to all-cause and cardiovascular mortality in southern Germany. Results from the MONICA Augsburg cohort study 1984-95. Ann Epidemiol 2002; 12: $338-45$

11 Yusuf S, Hawken S, Ounpuu S et al. Effect of potentially modifiable risk factors associated with myocardial infarction in 52 countries (the INTERHEART study): case-control study. Lancet 2004; 364: 937-952

12 Rosengren A, Hawken S, Ounpuu S et al. Association of psychosocial risk factors with risk of acute myocardial infarction in 11119 cases and 13648 controls from 52 countries (the INTERHEART study): casecontrol study. Lancet 2004; 364: 953 - 962

${ }^{13}$ Orth-Gomer K, Unden Al, Edwards ME. Social isolation and mortality in ischemic heart disease: a 10-year follow-up study of 150 middleaged men. Acta Med Scand 1988; 224: 205 -215

${ }^{14}$ Holohan CJ, Holohan CK, Moos RH et al. Social support, coping, and depressive symptoms in a late-middle-aged sample of patients reporting cardiac illness. Health Psychol 1995; 14: $152-163$

${ }^{15}$ Brummett BH, Barefoot JC, Siegler IC et al. Characteristics of socially isolated patients with coronary artery disease who are at elevated risk for mortality. Psychosom Med 2001; 63: 267-272

${ }^{16}$ Kubzansky LD, I Kawachi, A Spiro III et al. Is worrying bad for your heart? A prospective study of worry and coronary heart disease in the Normative Aging Study. Circulation 1997; 95: 818-824

${ }^{17}$ Haines AP, Imeson JD, Meade TW. Phobic anxiety and ischaemic heart disease. Brit Med J 1987; 295: 297-299
${ }^{19}$ Pignone MP, Gaynes BN, Rushton JL et al. Screening for depression in adults: a summary of the evidence for the U.S. Preventive Services Task Force. Ann Intern Med 2002; 136: 765 - 776

${ }^{20}$ Blazer DG, Kessler RC, McGonagle KA et al. The prevalence and distribution of major depression in a national community sample: the National Comorbidity Survey. Am J Psychiatry 1994; 151: 979-986

${ }^{21}$ Kessler RC, Berglund P, Demler O et al. The epidemiology of major depressive disorder: results from the National Comorbidity Survey Replication (NCS-R). JAMA 2003; 289: 3095 - 3105

22 Simon GE, von Korff M. Recognition, management, and outcomes of depression in primary care. Arch Fam Med 1995; 4: 99-105

${ }^{23}$ Murray CJL, Lopez AD. The Global Burden of Diseases: Summary. In: (ed) (Hrsg). Cambridge Mass: Harvard University Press, 1996

24 Rugulies R. Depression as a predictor for coronary heart disease. A review and meta-analysis. Am J Prev Med 2002; 23: 51 -61

${ }^{25}$ Wulsin LR, Singal BM. Do depressive symptoms increase the risk for the onset of coronary disease? A systematic quantative review. Psychosom Med 2003; 65: 259-267

${ }^{26}$ Ladwig KH, Erazo N, Rugulies R. Das Konzept der vitalen Erschöpfung, Angst und Depression, Hilf- und Hoffnungslosigkeit vor Ausbruch der koronaren Herzerkrankung. Reihe Statuskonferenz Psychokardiologie. Frankfurt/M.: VAS-Verlag, 2004

${ }^{27}$ Wassertheil-Smoller S, Shumaker S, Ockene J et al. Depression and cardiovascular sequelae in postmenopausal women. The Women's Health Initiative (WHI). Arch Intern Med 2004; 164: 289-298

${ }^{28}$ Ladwig KH, Marten-Mittag B, Baumert J et al. Case-finding for vital exhaustion and depressive mood in the general population: reliability and validity of a symptom driven diagnostic scale. Results from the MONICA Augsburg Study. Ann Epidemiol 2004; 14: 332 - 338

${ }^{29}$ Ladwig KH, Löwel H. Psychoimmunologische Folgen akuter und chronischer Stress-Belastung: Implikationen für die psychokardiologische Stressforschung. Rauch, B. Heldt R: Der multimorbide Patient. Heidelberg: Steinkopff Verlag, 2001: 161 - 168

${ }^{30}$ Ladwig KH, Marten-Mittag B, Löwel H et al. C-reactive protein, depressed mood and the prediction of coronary heart disease in initially healthy men. Eur Heart J (submitted)

${ }^{31}$ Ladwig KH, Marten-Mittag B, Löwel H et al. Influence of depressive mood on the association of CRP and obesity in 3,205 middle aged healthy men. Results from the MONICA Augsburg Study. Brain Behav Immun 2003; 17: $268-275$

32 Ladwig KH, Baumert J, Löwel $\mathrm{H}$ et al. Contemplating to quit current smoking status: Differences in behavioural and psychosocial patterns in a population-based cohort of current smokers. Prev Med 2005; 41: $134-140$

${ }^{33}$ von Kanel R, Mills PJ, Fainman C et al. Effects of psychological stress and psychiatric disorders on blood coagulation and fibrinolysis: a biobehavioral pathway to coronary artery disease? Psychosom Med 2001; 63: $531-544$

${ }^{34}$ Kop WJ, Gottdiener JS, Tangen CM et al. Inflammation and coagulation factors in persons $>65$ years of age with symptoms of depression but without evidence of myocardial ischemia. Am J Cardiol 2002; 89: $419-424$

${ }^{35}$ Bluthe RM, Michaud B, Poli V et al. Role of IL-6 in cytokine-induced sickness behavior: A study with IL-6 deficient mice. Physiol Behav 2000; 70: $367-373$

${ }^{36}$ Kaplan GA, Camacho T. Perceived health and mortality: a nine-year follow-up of the human population laboratory cohort. Am J Epidem 1983; 117: $292-304$

37 Spielberger CD, Gorsuch RL, Lushene RE. Manual for the State-Trait Anxiety Inventory. Palo-Alto: Consulting Psychologists Press 1970

38 Von Zerssen D. Die Beschwerden-Liste. Klinische Selbstbeurteilungsfragebögen aus dem Münchner Psychiatrischen Informationssystem. Psychis-Manuale, Allgemeiner Teil. Weinheim: Beltz, 1976

${ }^{39}$ Ware J, Kosinski M, Keller SD. Physical and mental component summary scales: a user's manual. The Health Institute, New England Medical Center. Boston, 1994

${ }^{40}$ Jenkins CD, Zyzanski SJ, Rosenman RH. Progress toward validation of a computer-scored test for the type A coronary-prone behavior pattern. Psychosom Med 1971; 33: $193-202$ 
${ }^{41}$ Karasek RA, Theorell TG, Schwartz J et al. Job, psychological factors and coronary heart disease. Swedish prospective findings and US prevalence findings using a new occupational inference method. Adv Cardiol 1982; 29: $62-67$

42 Siegrist J, Dittmann K, Rittner K et al. The social context of active distress in patients with early myocardial infarction. Soc Sci Med 1982; 16: $443-453$

43 Berkman LF, Syme SL. Social networks, host resistance, and mortality: a nine year follow-up study of Alameda County residents. Am J Epidemiol 1979; 109: 186-204

${ }^{44}$ Seeman TE, Kaplan GA, Knudsen L et al. Social network ties and mortality among the elderly in the Alameda County. Am J Epidemiol 1987; 126: $714-723$

${ }^{45}$ WHO . MONICA Psychosocial Optional Study MOPSY. Suggested measurement instruments [EUR/ICP/NCD 011, 3037H]. Copenhagen: WHO Regional Office for Europe, 1989

${ }^{46}$ Lowe B, Kroenke K, Herzog W et al. Measuring depression outcome with a brief self-report instrument: sensitivity to change of the Patient Health Questionnaire (PHQ-9). J Affect Disord 2004; 81: 61 - 66

47 Ware J, Kosinski M, Bayliss MS et al. Comparison of methods for the scoring and statistical analysis of SF-36 Health profile and summary measures: summary of results from the Medical Outcome Study. Med Care 1995; 33: 264

48 Bullinger M, Kirchberger I. SF-36 Fragebogen zum Gesundheitszustand. Göttingen: Hogrefe, 1998

${ }^{49}$ Zigmond AS, Snaith RP. The hospital anxiety and depression scale. Acta Psychiatr Scand 1983; 67: $361-370$

${ }^{50}$ Denollet JK. Type D personality and vulnerability to chronic disease, impaired quality of life (QOL), and depressive symptoms. Psychosom Med 2002; 64: 101

${ }^{51}$ Schumann A, Rumpf HJ, Meyer C et al. Deutsche Version des Fagerström-Test for Nicotine Dependence (FTND-G) und des Heaviness of Smoking Index (HSI-G). In: Glöckner-Rist A, Rist F, Küfner H (Hrsg). Elektronisches Handbuch zu Erhebungsinstrumenten im Suchtbereich (EHES). Version 3.00. Mannheim: Zentrum für Umfragen, Methoden und Analysen, 2003

52 Wallston KA, Wallston BS, DeVellis R. Development of the multidimensional health locus of control (MHLOC) scales. Health Educ Monogr 1978; 6: 160 - 170

${ }^{53}$ Janßen C, Heinrich C, Weitkunat R et al. Sozio-ökonomischer Status und gesundheitliche Kontrollüberzeugungen. Z Gesundheitswissenschaften 2000; 8: 216-229

${ }^{54}$ Fydrich T, Sommer G, Brähler E. Fragebogen zur Sozialen Unterstützung (F-SozU). Göttingen: Hogrefe, (in press)

55 Veenstra G. Social capital, SES and health: an individual-level analysis. Soc Sci Med 2000; 50: 619-629

${ }^{56}$ Siegrist J, Dittmann K, Rittner K et al. The social context of active distress in patients with early myocardial infarction. Soc Sci Med 1982; $16:: 443-453$

${ }^{57}$ Holle R, Happich M, Löwel H et al. KORA - A research platform for population based health research. Gesundheitswesen 2005; 67 S1: S19-S25

${ }^{58}$ Wichmann HE, Gieger C, Illig T et al. KORA-gen - Resource for population genetics, controls and a broad spectrum of disease phenotypes. Gesundheitswesen 2005; 67 S1: S26-S30

${ }^{59}$ Löwel H, Meisinger C, Heier M et al. The population-based Acute Myocardial Infarction (AMI) Registry of the MONICA/KORA study region of Augsburg. Gesundheitswesen 2005; 67 S1: S31 - S37

${ }^{60}$ Lederbogen F, Deuschle M für die KORA-Studiengruppe. Dysfunktion des Hypothalamus-Hypophysen-Nebennierenrinden-Systems - Hintergrundvariable relevanter kardiovaskulärer Risikofaktoren? Beschreibung eines kooperativen Projektes. Gesundheitswesen 2005; 67 S1: S94-S97

${ }^{61}$ Berger K, Roesler A, Kretzschmar K. The association between white matter lesions, stroke and activities of daily living - The MEMO Study on the KORA Platform Augsburg. Gesundheitswesen 2005; 67 S1: S172-S175

62 Braune BT, Berger K. The influence of depressive mood on activities of daily living and health care utilization in the elderly - The MEMO study on the KORA Platform Augsburg. Gesundheitswesen 2005; 67 S1: S176-S179

${ }^{63}$ Wildner M, Döring A, Meisinger C et al. Frakturen im höheren Lebensalter - eine Herausforderung für Prävention und Gesundheitsförderung - Ergebnisse der KORA-Frakturstudie Augsburg. Gesundheitswesen 2005; 67 S1: S180-S186 